\title{
No more wasting and loss of food products!
}

With the World Food Summit just ended in Rome and the Copenhagen Conference on the environment in full swing, my attention was drawn by an article in the scientific magazine PLOS One. It points out to the enormous waste of food on our planet today, while more than a billion people suffer from hunger. Not to mention that producing this food, which ends up wasted, requires huge amounts of freshwater and energy.

Who mentioned this in Rome and who is mentioning it in Copenhagen? As a matter of fact the phenomenon does not only affect industrialized countries. It also affects developing countries, in the form of losses rather than waste. Perishable products, in particular fruit and vegetables, are obviously on top of the list in terms of levels of losses.

Prevailing thought has it that an increase in agricultural production is necessary to meet food demand in the decades to come. Would it not be more rational to first seek to reduce waste and losses, which would in turn lower the pressure on resources and the environment?

Unfortunately, while the issue is increasingly put forward by researchers and specialized agencies, it still does not appear on the agenda of political priorities of most States, the very same ones deciding for the future of our planet in Rome and Copenhagen.

Beyond our strict role of scientists, we must seek to reach out for this scientifically proven evidence to be included in Summit declarations, whether on agriculture, food or the environment.

Dr. Jacky Ganry Chairman of the Section on Tropical and Subtropical Fruits ISHS 


\section{Éditorial}

\section{Halte au gaspillage et aux pertes des produits alimentaires !}

Alors que vient de se terminer le Sommet mondial sur l'alimentation à Rome, et que la Conférence de Copenhague sur l'environnement battait son plein, mon attention a été attirée par un article de la revue scientifique PLOS One. Celui-ci pointe du doigt l'énorme gaspillage de nourriture qui concerne aujourd'hui notre planète alors que plus d'un milliard de personnes souffrent de la faim. Ce gâchis alimentaire entraîne en plus un gaspillage énorme d'eau douce ainsi que d'énergie nécessaire à produire ces produits finalement non utilisés.

Qui a parlé de ce sujet à Rome et qui en a parlé à Copenhague? Car le phénomène ne concerne pas seulement les pays riches. Il touche aussi les pays en développement où l'on parle plus de pertes que de gaspillage. Les produits naturellement périssables figurent évidemment en tête de liste des produits agricoles ayant les plus hauts niveaux de perte et parmi eux les fruits et légumes sont parmi les plus concernés.

Alors que la pensée dominante veut qu'il faut augmenter la production agricole pour répondre à la demande alimentaire dans les décennies à venir, ne serait-il pas plus rationnel de chercher d'abord à réduire le gaspillage et les pertes, ce qui permettrait aussi de réduire la pression sur les ressources et l'environnement?

Malheureusement, si le sujet est de plus en plus mis en avant par les chercheurs et les institutions spécialisées, il n'est toujours pas à l'agenda des politiques prioritaires d'une grande majorité des États qui sont les mêmes que ceux qui décident pour l'avenir de notre planète à Rome ou à Copenhague.

Au-delà de notre strict rôle de scientifiques, nous devons nous préoccuper de faire entendre notre voix pour que de telles évidences prouvées scientifiquement figurent un jour dans les déclarations des sommets qu'ils soient sur l'agriculture, l'alimentation ou sur l'environnement.

Dr. Jacky Ganry

Président de la section Fruits tropicaux et subtropicaux 